\title{
Vier Nauklerosquittungen \\ (P.UB Trier S 84-1 bis 84-3b und 108-4)
}

\author{
Mit Tafeln II-V* \\ Stefan Pfeiffer (Trier)
}

Bekanntermaßen wurde das Geschäft der Verschiffung des Steuergetreides von Naukleroi betrieben, die für das ihnen anvertraute Getreide verantwortlich waren. Diese Verantwortung ist durch eine reiche Zahl an Nauklerosquittungen dokumentiert. Die im folgenden vorgestellten Papyri der Trierer Sammlung gehören dieser Textgruppe an. Alle stammen aus Mumienkartonage. Die ersten drei Quittungen wurden im Jahr 1985 erworben. Sie wurden etwa zur selben Zeit in der Mitte des 2. Jhs. v.Chr. im bekannten Hafen von Kaine im Polemon-Bezirk des Arsinoites ausgestellt. In allen drei Fällen bescheinigt ein jeweils anderer Naukleros Peteharpsenesis, dem Sitologen des Ergasterions von Tebtynis, die Verladung von Getreide für den königlichen Speicher in Alexandria. Die Arbeitsstelle des bekannten Sitologen und die Funktion von Kaine als Verladehafen für das Steuergetreide des Bezirkes von Tebtynis sind hier zum ersten Mal bezeugt. Die drei Trierer Papyri ergänzen auf diese Weise das Bild dieses Hafens am Rande des Faijums, das sich aus den P.Erasm. II ergeben hat. Auch begegnet hier zum ersten Mal der Schiffstyp Kerkuros mit einer Ladekapazität von 11000 Artaben (P.UB Trier S 84-2,8), der der Zusammenstellung von Poll hinzugefügt werden kann. ${ }^{1}$ Die vierte Quittung stammt aus einem anderen Kauf von 1989; sie gehört aber vielleicht in denselben Rahmen.

Zum Urkundentyp vgl. die grundlegende Arbeit von Ph.A. Verdult, P.Erasmianae II. Parts of the Archive of an Arsinoite Sitologos from the Middle of the Second Century BC, Amsterdam 1991 (P.Erasm. II); des weiteren D.G. Herring, New Ptolemaic Documents Relating to the Shipment of Grain: Five Naukleros Receipts and an Order to Sitologoi, ZPE 76, 1989, 27-37; W. Clarysse, H. Hauben, Ten Ptolemaic Granary Receipts from Pyrrheia, ZPE 89, 1991, 47-68; R. Duttenhöfer, Ptolemäische Urkunden aus der Heidelberger Papyrussammlung (P.Heid. VI), Heidelberg 1993, Nr. 369; M. Parca, Ptolemaic Administrative Correspondence Concerning a Shipment of Grain (P. Mich. Inv. 6992), in: CdÉ 77, 2002, 191-196.

\footnotetext{
* Digitalisierte Abbildungen der Trierer Papyri sind bereitgestellt unter <http://www.digipap. uni-trier.de>.

${ }^{1}$ I.J. Poll, Ladefähigkeit und Größe der Nilschiffe, APF 42, 1996, 135-138.
} 
Zur Kornsammlung und zum Korntransport vgl. E. Börner, Der staatliche Korntransport im griechisch-römischen Ägypten, Diss. Hamburg 1939; H. Hauben, Nouvelles remarques sur les nauclères d'Égypte. L'époque des Lagides, ZPE 28, 1978, 99-108; dens., Le transport fluvial en Égypte ptolémaïque. Les bateaux du roi et de la reine, Actes du XVe Congrès international de Papyrologie. Quatrième partie. Papyrologie documentaire, Brüssel 1979, S. 68-77; W. Kunkel, Verwaltungsakten aus spätptolemäischer Zeit, APF 8, 1927, 183-196; Cl. Préaux, L'économie royale des Lagides, Brüssel 1939, 129-137; 143-147; M. Rostovtzeff, The Social and Economic History of the Hellenistic World I, Oxford 1941, 280; 314-315; dens., Kornerhebung und -transport im griechisch-römischen Ägypten, APF 3, 1906, 201-224; A.J.M. Meyer-Termeer, Die Haftung der Schiffer im griechisch-römischen Recht, Zutphen 1978; U. Wilcken, Grundzüge und Chrestomathie der Papyruskunde. Erster Band: Historischer Teil. Erste Hälfte: Grundzüge, Berlin 1912, S. 376-380.

$\mathrm{Zu}$ den Schiffstypen vgl. L. Casson, Ships and Seamanship in the Ancient World, Princeton 1973; I.J. Poll, Ladefähigkeit und Größe der Nilschiffe (s.o. Anm. 1).

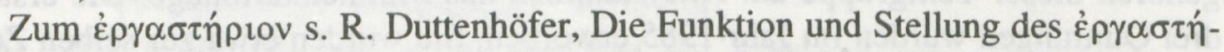
prov in der Getreideverwaltung der Ptolemäerzeit, ZPE 98, 1993, 253-262.

\section{Quittung des Dionysios für Peteharpsenesis}

P.UB Trier S 84-1

Kaine (Arsinoites)
$11,5 \times 21 \mathrm{~cm}$ Tafel II
29. Mai 154 v. Chr. Recto; Verso unbeschrieben

Der Text ist in einer kursiven Geschäftsschrift des 2. Jhs. v.Chr. parallel zur Faserrichtung geschrieben. Er setzt etwa 2 bis $2,5 \mathrm{~cm}$ vom linken Rand entfernt ein und reicht teilweise bis an den rechten Rand heran. Oben ist der Papyrus kurz oberhalb der Datumsangabe abgebrochen, unten mitten in den Angaben über den rechten Gebrauch des Kornmaßes. Wesentlich mehr wird dort aber auch nicht gestanden haben, denn aus dem Vergleich mit anderen Nauklerosquittungen ergibt

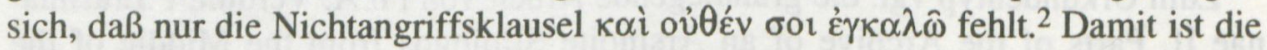
Außenschrift (scriptura exterior), also die ungekürzte Quittung selbst, nahezu komplett erhalten, wohingegen die in solchen Dokumenten zu erwartende Innenschrift (scriptura interior), also die versiegelte Kurzfassung des Inhalts, fehlt. ${ }^{3}$

Auffällig ist der inkonsequente Gebrauch des t-adscriptum in Z. 16-17, der insbesondere für die Mitte des zweiten vorchristlichen Jahrhunderts gut belegt ist. ${ }^{4}$

Der Inhalt der Quittung läßt sich wie folgt zusammenfassen: Ein Naukleros namens Dionysios, der eine Prosagogis des Herakleides fährt, erklärt, daß er vom

2 Vgl. die Nauklerossymbola P.Erasm. II 36, 38, 39, 40, 41.

3 Vgl. die Beschreibung bei Duttenhöfer, P.Heid. VI, S. 53; Verdult, P.Erasm. II., S. 123.

${ }^{4}$ Verdult, P.Erasm. II, S. 4; s. auch W. Clarysse, Notes on the Use of the Iota Adscript in the Third Century B.C., CdÉ 51, 1976, 105-166, besonders 160 . 
Sitologen Peteharpsenesis im Hafen von Kaine 350 Artaben Steuerweizen als Ladung erhalten hat. Diese wird er in den Königlichen Speicher nach Alexandria bringen. In P.Erasm. II 36-58 steht uns eine große Menge von Paralleltexten aus der gleichen Zeit und auch vom gleichen Verladehafen zur Verfügung; zu Einzelveröffentlichungen dieses Urkundentyps vgl. o. die in der Einleitung angegebene Literatur, insbesondere die zehn von W. Clarysse und H. Hauben publizierten Nauklerossymbola.

Paläographisch weist der Text, wie bereits gesagt, in das 2. Jh. v.Chr. Weiter eingrenzen läßt sich die Abfassungszeit durch die Angabe ,am 1. Pachon des 27. Regierungsjahres". Nur zwei Könige zählen in der angegebenen Zeit 27 Regierungsjahre und mehr, Ptolemaios VI. zusammen mit Kleopatra II. (36 Jahre) und Ptolemaios VIII. (54 Jahre). ${ }^{5}$ Im zweiten Falle wäre das Datum des Papyrus der 26. Mai 143 v.Chr. Da jedoch die enge Parallele Nr. 2 (P.UB S 84-2) die ausführ-

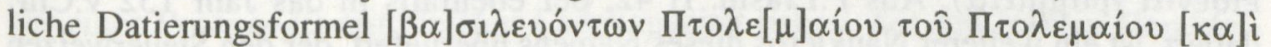

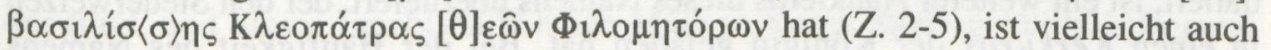
der vorliegende Text eher in die Regierungszeit Ptolemaios' VI. und Kleopatras II. zu datieren. Damit ergibt sich als Abfassungsdatum der 29. Mai 154 v.Chr.

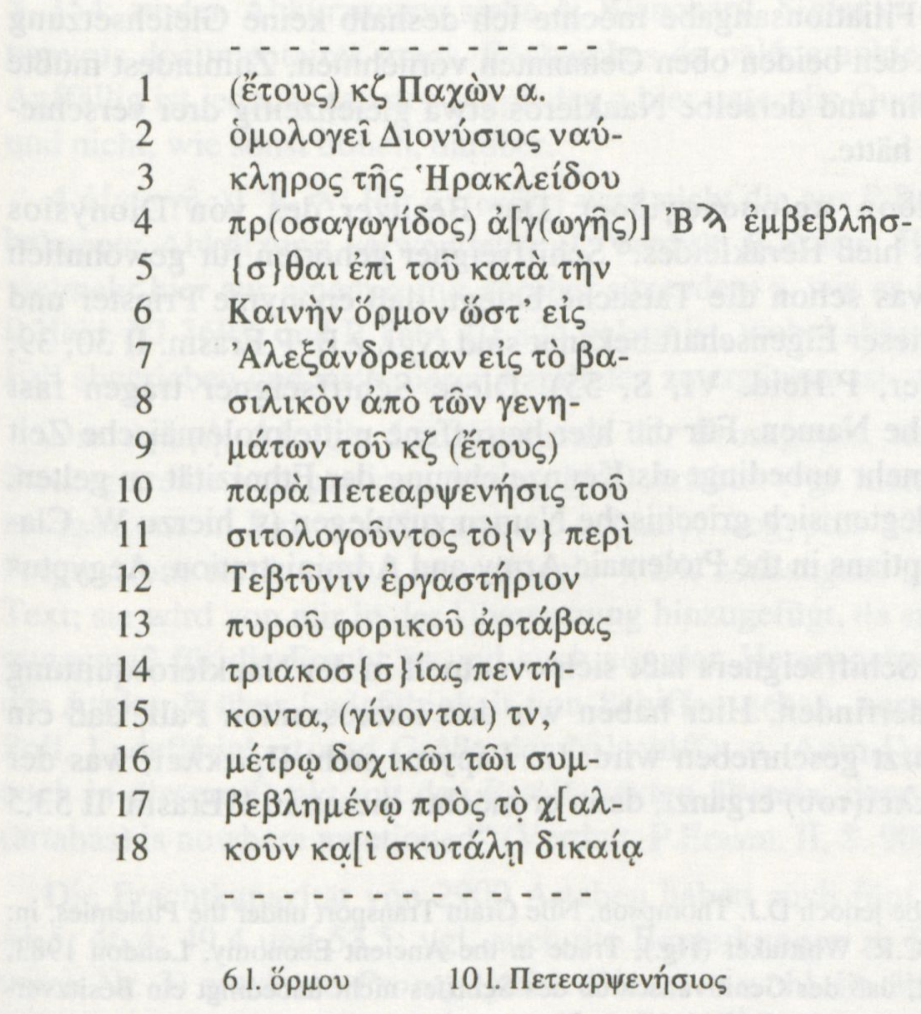

${ }^{5}$ Vgl. Th.C. Skeat, The Reigns of the Ptolemies, München ${ }^{2} 1969$, S. 14-15. 


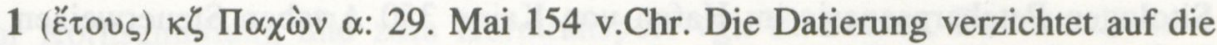
Angabe der regierenden Herrscher, was sich bei einer Durchsicht der überlieferten Nauklerosquittungen als relativ häufig erweist. Ein Strich, der das $\alpha$ als Tagesdatum kennzeichnen könnte, ist nicht sichtbar; entweder hat es ihn nie gegeben oder er ist mit dem oberen Rand weggebrochen.

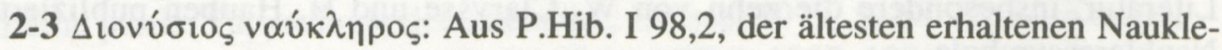
rosquittung aus dem Jahr 251 v.Chr., ist ein Naukleros namens Dionysios bekannt, der sich aus chronologischen Gründen selbstverständlich nicht mit unserem Dionysios in Verbindung bringen läßt. Jedoch ist für das Jahr $152 \mathrm{v}$.Chr. ebenfalls ein Naukleros dieses Namens belegt (P.Erasm. II 36,4), der einen Kerkuros von Archedemos und Andromachos für den Korntransport nutzt. Von diesem Dionysios ist noch zu erfahren, daß er nicht schreiben konnte (P. Erasm. II 36,27: $\mu \grave{\eta}$

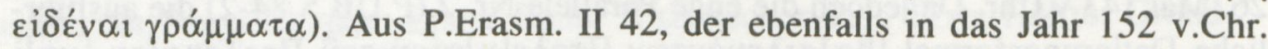
datiert, ist ein weiterer Naukleros dieses Namens überliefert, der den Steuerweizen in einen Kerkuros der Königin geladen hat. Bei der Häufigkeit des Namens Dionysios in dieser Zeit zögert Verdult zu Recht, die beiden letztgenannten Naukleroi miteinander zu identifizieren (P.Erasm. II, S. 68, Anm. 1). Aus demselben Grund ist auch bei dem neuen, zwei Jahre älteren Beleg Vorsicht angebracht. Ohne weitere Filiationsangabe möchte ich deshalb keine Gleichsetzung unseres Dionysios mit den beiden oben Genannten vornehmen. Zumindest müßte man annehmen, daß ein und derselbe Naukleros etwa gleichzeitig drei verschiedene Schiffe betrieben hätte.

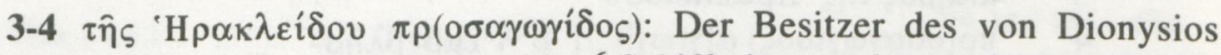
genutzten Frachtkahns hieß Herakleides. ${ }^{6}$ Schiffseigner gehörten für gewöhnlich der Oberschicht an, was schon die Tatsache belegt, daß eponyme Priester und sogar die Königin in dieser Eigenschaft bekannt sind (vgl. z.B. P.Erasm. II 30; 39; 42; 43; s. Duttenhöfer, P.Heid. VI, S. 55). Diese Schiffseigner tragen fast durchgängig griechische Namen. Für die hier betroffene mittelptolemäische Zeit hat dies jedoch nicht mehr unbedingt als Kennzeichnung der Ethnizität zu gelten, denn auch Ägypter pflegten sich griechische Namen zuzulegen (s. hierzu W. Clarysse, Greeks and Egyptians in the Ptolemaic Army and Administration, Aegyptus $65,1985,57-66)$.

Der Name unseres Schiffseigners läßt sich eventuell in der Nauklerosquittung P.Erasm. II 53,5 wiederfinden. Hier haben wir den interessanten Fall, daß ein Personenname abgekürzt geschrieben wird. Im Papyrus steht $\mathrm{H} \rho \alpha \kappa \lambda \varepsilon$, was der

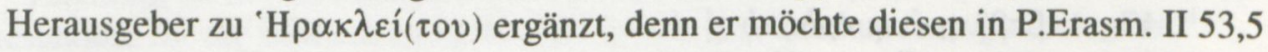

${ }^{6}$ Vgl. zur Besitzerangabe jedoch D.J. Thompson, Nile Grain Transport under the Ptolemies, in: P. Garnsey, K. Hopkins, C.R. Whittaker (Hg.), Trade in the Ancient Economy, London 1983, S. 67, die der Meinung ist, daß der Genitivanschluß des Schiffes nicht unbedingt ein Besitzverhältnis ausdrücken muß. Duttenhöfer, P.Heid. VI, S. 55, Anm. 5, führt diese Meinung an, ohne sie jedoch weiter zu kommentieren. Da Thompson keine wirkliche Alternative zur bisherigen Deutung bieten kann, möchte ich hier an der traditionellen Bestimmung des Genitivs als Schiffseignerangabe festhalten. 
erwähnten Schiffseigner mit einem aus P.Lille I 21,7 (155/144 v.Chr. = PP V

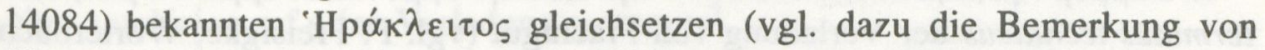
Clarysse und Hauben, ZPE 89, 1991, 56 [Kommentar zu P.Lille I 21]: ,the name

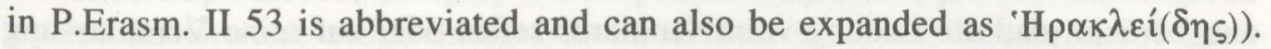
Verdult hatte eine Ergänzung des Namens zu Herakleides in Erwägung gezogen, doch wieder verworfen, weil ein ebenfalls als Schiffseigner bekannter Herakleides (P.Tebt III 825 = PP V 14082) aus dem Jahre 176 v.Chr. stammt und danach seiner Meinung nach zeitlich zu weit entfernt von P.Erasm. II 53 gelebt hätte (S. 60: „he must have been very old in $149 \mathrm{BC}$ "). Die vorliegende Nauklerosquittung aus unmittelbarer zeitlicher Nähe legt aber nahe, daß der Eigner der Prosagogis von

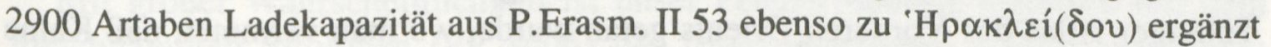
werden kann, denn auch unser Herakleides hatte eine Prosagogis von gleichem Frachtvolumen. Da wir also für die Jahre 154 und 149 v.Chr. zweimal einen Eigner einer Prosagogis mit gleicher Ladekapazität haben, der den Namen Herakleides trägt, und dieses Frachtschiff beide Male in Kaine Steuergetreide aufnimmt, ist mit hoher Wahrscheinlichkeit eine Identität beider Personen anzunehmen.

Die Schiffsbezeichnung Prosagogis ist mit einem Monogramm geschrieben, wie es auch in P.Erasm. II 46,4 und 53,5 vorkommt (vgl. Verdult, P.Erasm. II, S. 154; zu den Abkürzungen siehe A. Blanchard, Sigles et abbréviations dans les papyrus documentaires grecs, Recherches de paléographie, London 1974, S. 4). Auffällig ist jedoch, daß der 'Kopf' des $\rho$ hier unter die Querhaste des $\pi$ gesetzt ist und nicht, wie sonst üblich, darüber.

$4 \dot{\alpha}[\gamma(\omega \gamma \hat{\eta} \varsigma)]$ 'B $\lambda$ : Für $\dot{\alpha} \gamma(\omega \gamma \hat{\eta} \varsigma)$ wird nicht die aus P.Erasm. II 25,9 und 28,7 bekannte Abkürzung verwendet (vgl. Verdult, P.Erasm. II, S. 155). Sie besteht vielmehr hier aus einem $\alpha$ mit darüber sitzendem $\gamma$, wie es in P.UB. Trier S 84-2, P.Heid. VI 369,6 und P.Tebt. III 824 belegt ist, wobei aber das $\gamma$ im vorliegenden Fall abgerieben und nach diesen Parallelen zu ergänzen ist.

Die $\grave{\alpha} \gamma \omega \gamma \eta \dot{,}$ d.h. die Ladekapazität der Prosagogis, mit der Dionysios das Steuergetreide transportierte, betrug 2900 Artaben (vgl. hierzu M. Merzaggora, La

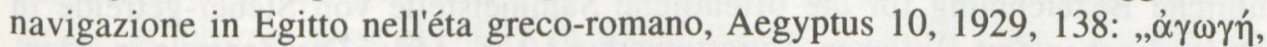
l'espressione tecnica per il tonnellaggio“). Die Maßangabe „Artabe“ steht nicht im Text; sie wird von mir in der Übersetzung hinzugefügt, da sie das übliche Berechnungsmaß für die Fracht ist und auch von den Herausgebern anderer Urkunden, die Auskunft über Ladefähigkeit von Schiffen geben, vorausgesetzt wird (siehe Poll, Ladefähigkeit und Größe der Nilschiffe, o. Anm.1). Der Papyrus stimmt auch in diesem Punkt mit den Paralleltexten überein, denn ,the unit of measure (artabas) is nowhere mentioned" (Verdult, P.Erasm. II, S. 90).

Die Frachtkapazität von 2900 Artaben haben auch fünf in P.Erasm. II $(38,4$; 41,$5 ; 46,4 ; 49,4$ und 53,5; vgl. auch die Bemerkungen zu P.UB Trier S 84-3b,5, unten Nr. 3) erwähnte Prosagogides. Dies war wohl die übliche Tonnage dieses Schiffstyps. In seiner Zusammenstellung gibt Poll, Ladefähigkeit und Größe der Nilschiffe (o. Anm.1), nur einen weiteren Beleg, demzufolge eine Prosagogis mehr, genauer 3500 Artaben, laden kann (P.Lille I 21,8; 2. Jh. v.Chr.). 


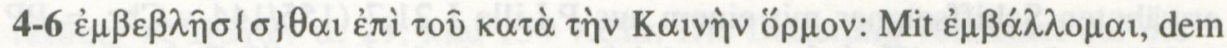
terminus technicus der Verladung von Frachtgut (vgl. F. Preisigke, Wörterbuch der griechischen Papyrusurkunden I, Sp. 472; Verdult, P.Erasm. II, S. 3), wird ausgedrückt, daß die Ladung vom Naukleros selbst oder auf seine Veranlassung hin an Bord verfrachtet worden ist (Meyer-Termeer, Die Haftung der Schiffer, S. 77, Nr. 4). Der archäologisch noch nicht nachgewiesene Hafen Kaine ist aus dem Sitologenarchiv P.Erasm. II reich belegt. Auch hier erhalten die Naukleroi ihr Getreide an diesem Umschlaghafen. Verdult, P.Erasm. II, S. 7, gibt die mutmaßliche Lage des Hafens auf einer Karte des Arsinoitischen Gaues an. Er sucht ihn in der Nähe von Krokodilon Polis, also um einiges weiter nördlich, als dies im Tübinger Atlas des Vorderen Orients getan wurde, wo man vermutet, daß Kaine nördlich von Herakleopolis am Bahr Jussuf gelegen habe (TAVO B V 21). A. Calderini und S. Daris, Dizionario dei nomi geografici e topografici dell'Egitto greco-romano III, Mailand 1978, S. 48, Nr. 3 und Supplemento 1, Mailand 1988, S. 161, hatten die Urkunden des Sitologenarchivs P.Erasm. II noch nicht gekannt. Weitere Belege für den Hafen nennt auch Duttenhöfer, P.Heid. VI, S. 56.

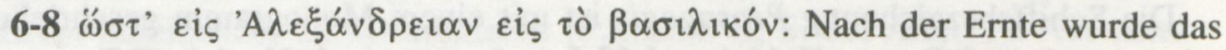
Steuergetreide in den Getreidespeichern der ägyptischen Chora gesammelt, um dann zum größten Teil, wie auch im vorliegenden Fall, nach Alexandria in den königlichen Speicher ( $\theta \eta \sigma \alpha v \rho o ́ \varsigma)$ gebracht zu werden (Wilcken, Chrestomathie I, 1, S. 376; vgl. auch die anschauliche Beschreibung des Vorganges bei Rostovtzeff, The Social and Economic History I, S. 280 oder Thompson, Nile Grain Transport, in: Garnsey, Hopkins, Whittaker [Hg.], Trade, S. 65). Die Sitologen der Ergasterien, die bis zum Verladehafen in der Verantwortung für das Korn standen, übergaben dieses hier an die Naukleroi, die seinen Transport nach Alexandria besorgten. Das Wort $\beta \alpha \sigma \imath \lambda \iota$ kóv wird in den Editionen von Nauklerosquittungen immer mit „königlicher Kornspeicher“ übersetzt (vgl. Duttenhöfer, P.Heid. VI, S. 55; Wilcken, Chrestomathie I, 1, S. 376; Clarysse und Hauben, Ten Ptolemaic granary receipts, ZPE 89, 1991, 56; Verdult, P.Erasm. II., S. 25 u.a.). Es ist die übliche Bezeichnung für die öffentliche Sammelstelle von Steuer-getreide in ptolemäischer Zeit (Wilcken, Chrestomathie I,1, S. 153: „Die Zentrale in Alexandrien hieß auch für das Naturaldepartement $\tau$ ò $\beta \alpha \sigma \iota \lambda$ ıкóv").

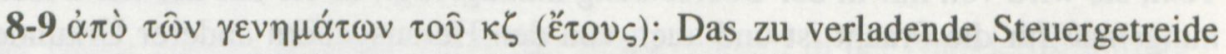
stammt aus dem 27. Regierungsjahr und ist damit frisch geerntet, denn die Quittung datiert auf dasselbe Jahr. Dies scheint die für die Ptolemäerzeit übliche Praxis gewesen zu sein (vgl. Verdult, P.Erasm. II, S. 4). Mit dem ersten Pachon, an dem die Transaktion stattfand, befand man sich noch mitten in der Erntezeit, denn in der Mitte des zweiten Jahrhunderts v.Chr. waren die Erntemonate der Pharmuthi und der Pachon, was dem Zeitraum von Mai bis Juni entspricht (Verdult, P.Erasm. II, S. 5; vgl.: W. Clarysse, Harmachis, Agent of the Oikonomos: An Archive from the Time of Philopator, AncSoc 7, 1976, 187). Das Steuergetreide 
kam somit direkt von der Tenne und hatte noch nicht allzu lange im Getreidespeicher von Kaine auf seine Verladung gewartet. ${ }^{7}$

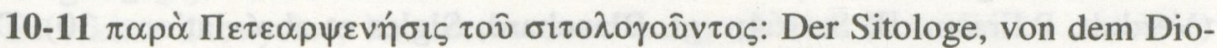
nysios das Getreide ausgehändigt bekommen hat, ist seinem Namen nach eindeutig als Ägypter zu identifizieren. Es handelt sich um die griechische Wiedergabe des ägyptischen Namens P3-tj-hrr-p3-šr-Is.t, der demotisch gut belegt ist (E. Lüddeckens und H.J. Thissen, Demotisches Namenbuch I, 1, Wiesbaden 2000, S. 329) und heißt übersetzt „Der, den 'Horus, der Sohn der Isis' gegeben hat“ (H. Ranke, Die ägyptischen Personennamen I, Glückstadt 1935, S. 125, Nr. 1).

Mit großer Wahrscheinlichkeit ist unser Peteharpsenesis identisch mit dem gleichnamigen Sitologen (PP 1406) der fragmentarischen Nauklerosquittung P.Tebt. III 2, 1034. Mit Hilfe des vorliegenden Papyrus läßt sich diese jetzt ziemlich sicher auf die Zeit um 150 (und nicht 139) v.Chr. datieren. Auch in P.UB Trier S 78-5,1 ist ein nicht weiter gekennzeichneter Peteharpsenesis Adressat eines amtlichen Schreibens.

Die beiden anderen Zeitgenossen dieses Namens kommen m.E. nicht in Betracht, weder der Königsbauer aus P.Tebt. III 2, 1003,11 vom 22.3.176 oder 19.3. 165 v.Chr. (vgl. H. Cuvigny, L'arpentage par espèces dans l'Égypte ptolémaïque d'après les papyrus grecs, Brüssel 1985, S. 47-50), noch der Königliche Schreiber aus P.Amh. II 33 und 34, [beide um 157 v.Chr.] = PP I 463; P.Tebt. III 2, 936 (?) [um 155 v. Chr.]), da es weder für den Aufstieg vom Königsbauern zum Sitologen noch für den eines Sitologen zum Königlichen Schreiber Belege gibt.

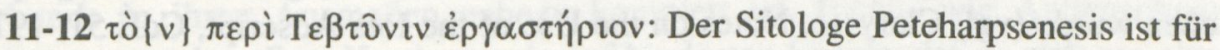
das Ergasterion in Tebtynis (Calderini und Daris, Dizionario IV, S. 377-382 und Supplemento 2, S. 209-210) zuständig. Diese nur für den arsinoitischen Gau belegte Institution trägt ,den langen Transportwegen zum Nil Rechnung“; hier wurde das Steuergetreide des zuständigen Gebietes zentral erfaßt (Duttenhöfer, Die Funktion und Stellung, in: ZPE 98, 1993, 260). Dies erklärt, weshalb Peteharpesenesis in den gleichzeitigen Nauklerosquittungen der P.Erasm. II nicht auftaucht, obwohl diese ebenfalls in Kaine ausgestellt worden sind. Die dort erwähnten Sitologen waren zum größten Teil im Ergasterion von Oxyrhyncha tätig (vgl. Verdult, P.Erasm. II, S. 3) und nicht in dem von Tebtynis. Gibt Duttenhöfer (P.Heid. VI, S. 56) noch an, daß es nicht sicher sei, „ob Kaine auch aus anderen Ergasterien mit Getreide beliefert wurde“, so läßt sich mit unserem Papyrus belegen, daß auch das Getreide aus Tebtynis zur Verschiffung nach Alexandrien hierhin gebracht wurde. Für beide Ergasteria, die, wie zumindest die Karte bei Verdult (P.Erasm. II, S. 7) zeigt, nicht weit voneinander entfernt lagen, war Kaine der Verladehafen.

7 Getreide wurde das gesamte Jahr über aus den Kornspeichern der Gaue nach Alexandria verschifft; die unmittelbare zeitliche Nähe zur Ernte läßt somit keine weiteren Schlüsse über den Zusammenhang von Getreidesteuer und Zeitpunkt der Verschiffung nach Alexandria zu (vgl. Thompson, Nile Grain Transport, in: Garnsey, Hopkins, Whittaker (Hg.), Trade, S. 73-74). 


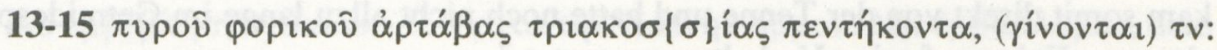
Der Abkürzungsstrich für $\gamma^{\prime}$ ivov $\tau \alpha_{\imath}$ ist ebenso wie in den P.Erasm. II geschrieben (Verdult, P.Erasm. II, S. 164; vgl. Blanchard, Sigles et abréviations, 30). Daß hinter den Zahlzeichen $\tau v$ keine Wiederaufnahme der Maßeinheit und des gemessenen Produktes erscheint, ist üblich und folglich nicht zu erwarten.

Der überwiegende Teil der bekannten Nauklerosquittungen hat die Verladung von Steuergetreide zum Inhalt (vgl. Verdult, P.Erasm. II, S. 3). Obwohl in einigen nur $\pi$ vó́ $\varsigma$ ohne Zusatz steht, kann es sich Verdult zufolge dabei nur um $\pi$ vò̀ $\varsigma$ popıкó handeln (Verdult, P.Erams. II, S. 3 mit weiteren Literaturangaben zu

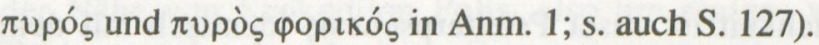

Interessant ist, daß der Naukleros mit der Verladung von 350 Artaben Steuergetreide die Ladekapazität des Schiffes von 2900 Artaben nicht annähernd ausgenutzt hat. Es war aber durchaus üblich, daß ein Naukleros an mehreren Orten Ladung aufnahm (vgl. Thompson, Nile grain transport, in: P. Garnsey, K. Hopkins, C.R. Whittaker [Hg.], Trade, S. 66., mit Verweis auf P.Tebt. III 2, 856, 58-59). Eventuell erhielt Dionysios im selben Hafen auch noch Getreide aus dem Ergasterion von Oxyrhyncha. Auch wird er vielleicht noch andere Verladehäfen angelaufen haben, um die Kapazität des Schiffes voll auszunutzen.

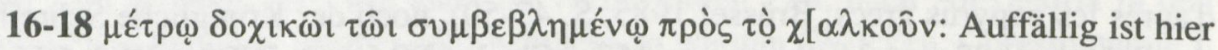
die inkonsequente Schreibung der Dative mit und ohne t-adscriptum. Der Schreiber scheint gewußt zu haben, daß man in korrekter Schreibweise üblicherweise ein 1 hinzufügen sollte, obwohl es im 2. Jh. v.Chr. längst nicht mehr gesprochen wurde (vgl. Clarysse, Notes on the Use of the Iota Adscript, CdÉ 51, 1976, 105-166). Daß er den Buchstaben im Dativ dann trotzdem zweimal weggelassen hat, zeigt seine Unsicherheit.

Die vorliegende Formel vom rechten Gebrauch des Maßes gehört zu den Grundbestandteilen einer Nauklerosquittung (vgl. Verdult, P.Erasm. II, S. 127), denn sie gewährte beiden Transaktionspartnern Rechtssicherheit in Bezug auf das verladene Frachtgut. Mit Sicherheit ist das Bronzemaß, mit dem das Korn abgemessen wurde, identisch mit dem des königlichen Speichers von Alexandria, so $\mathrm{da}$ eine Unterschlagung von Getreide beim Verladevorgang nicht möglich war. Die Formel $\mu \varepsilon \varepsilon \rho \omega \delta о \chi \iota \kappa \hat{\imath} \iota$ ist von Verdult untersucht worden (P.Erasm. II, S. 152-153). Er kommt zu dem Schluß, daß das ,metron dochikon in all likelihood measures out artabas of 36 choinikes" (P.Erasm. II, S. 153). Verdult setzt hier einen Umrechnungswert von $40 \mathrm{~kg}$ je Artabe an, was bedeuten würde, daß unser Naukleros 14 Tonnen Getreide in Kaine geladen hatte und seine Prosagogis eine Ladekapazität von 116 Tonnen besaß.

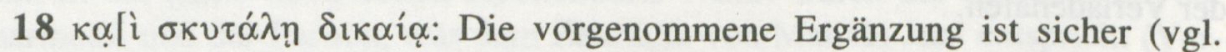
Verdult, P.Erasm. II, S. 124). Der Kontraktant versichert nicht nur, daß das Korn im richtigen $\mathrm{Ma} \beta$ verladen wurde, sondern auch, da $\beta$ das richtige Abstreichholz verwendet wurde (vgl. Wilcken, Chrestomathie I, 1, S. LXX; s. auch Verdult, P.Erasm. II, S. 153). 


\section{Übersetzung}

27. Regierungsjahr, 1. Pachon.

Es erklärt Dionysios, Naukleros der Prosagogis des Herakleides mit einer Ladekapazität von 2900 (Artaben), geladen zu haben im Hafen von Kaine mit dem Ziel des königlichen Speichers in Alexandria von den Erträgen des 27. Jahres von Peteharpsenesis, dem Sitologen des Ergasterions bei Tebtynis, dreihundertfünfzig Artaben Steuerweizen, macht 350, mit dem Empfangsmaß, das verglichen ist mit dem bronzenen Normalmaß und mit dem geraden Streichmaß ---

\section{Quittung des Apollonios für Peteharpsenesis}

P.UB Trier S 84-2

Kaine (Arsinoites)

$$
8 \times 29 \mathrm{~cm}
$$

Tafel III
1. September 155 v.Chr. Recto; Verso unbeschrieben

Der Papyrus besteht aus zwei Fragmenten, an deren unterer und oberer Bruchstelle nach Z. 18 der Text verloren ist. Oben ist ein 1,3 cm Rand sichtbar, rechts reicht die Schrift teilweise bis zum Rand, und auf der linken Seite ist der Papyrus schräg zurechtgeschnitten, sodaß das Blatt nach oben hin schmaler wird, wodurch in Z. 1 drei und in Z. 2-9 ein bis zwei Buchstaben fehlen. Auch die im Abstand von 4,2 cm über der Außenschrift angebrachte, den Quittungsinhalt zusammenfassende Innenschrift ist noch vorhanden, was genauso wie der $9 \mathrm{~cm}$ hohe unbeschriebene Freiraum unter dem Haupttext darauf hinweist, daß die Urkunde in ihren Ausmaßen nahezu komplett ist. Innen- wie Außenschrift stammen von derselben Hand, was darauf hindeuten könnte, daß es sich um eine Abschrift handelt. Das Verso trägt keine Beschriftung. Der Papyrus hat ein Loch zwischen Innen- und Außenschrift. Duttenhöfer (P.Heid. VI, S. 53) hat die Funktion dieses Loches beschrieben: Üblicherweise wurde der obere Teil des Papyrus mit der Innenschrift eingerollt, mit Hilfe eines Papyrusstreifens, der durch das Loch zwischen Innen- und Außenschrift geführt wurde, verschnürt und in diesem Zustand aufbewahrt. So weist das auf den ursprünglichen Aufbewahrungszustand des Textes hin (vgl. die Abbildung einer zusammengerollten Innenschrift bei P.W. Pestman, Greek and Demotic Texts from the Zenon Archive. Plates, Leiden 1980, Taf. I, oben links).

Im Gegensatz zur geübten Kursive des oben besprochenen P.UB Trier S 84-1 (Nr. 1) wirkt die Schrift des vorliegenden weniger geübt und etwas archaischer, da die Zeichen teilweise ohne Ligaturen nebeneinander gesetzt wurden.

Apollonios, der Naukleros eines Kerkuros, quittiert dem Sitologen Peteharpsenesis im Hafen von Kaine den Erhalt von 3000 Artaben Weizen. Es folgt eine lacuna im Umfang von drei Zeilen, worauf man am Ende nochmals die Mengenangabe „,70 Artaben Gerste“ lesen kann, die wahrscheinlich in Zusammenhang mit Aussagen über die Qualität des geladenen Getreides zu sehen ist.

Datiert ist die Quittung durch die Angabe des Regierungsjahres Ptolemaios' VI. Philometor und Kleopatras II. Da das in Nauklerosquittungen quittierte Getreide - 
immer im Jahr seiner Ernte verladen wurde, läßt sich in Z. 1 das 26. Regierungsjahr ergänzen. Somit wurde die vorliegende Nauklerosquittung am 1. September 155 v.Chr. ausgestellt.

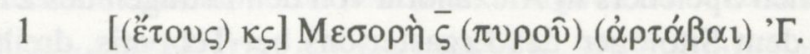

$2[\beta \alpha] \sigma i \lambda \varepsilon v o ́ v \tau \omega v \Pi \Pi \tau 0 \lambda \varepsilon-$

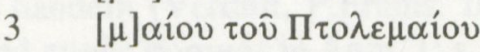

$4 \quad[\kappa \alpha] i \beta \alpha \sigma \imath \lambda i ́ \sigma\langle\sigma\rangle \eta \varsigma \mathrm{K} \lambda \varepsilon \circ \pi \alpha \dot{\tau} \tau \rho \alpha \varsigma$

$5[\theta] \varepsilon \hat{\omega} v \Phi ı \lambda o \mu \eta \tau o ́ \rho \omega v$

6 [ỏ]

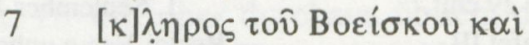

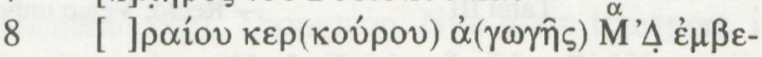

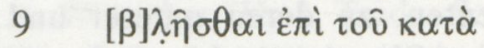

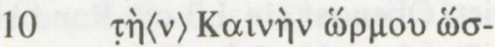

$11 \tau \varepsilon \varepsilon i \varsigma^{\prime} A \lambda \varepsilon \xi{ }^{\prime} \alpha{ }^{\prime} \delta \rho ! \alpha v$

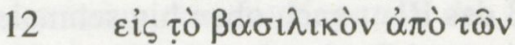

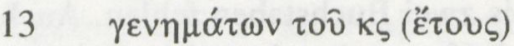

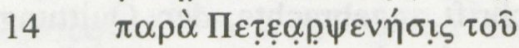

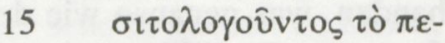

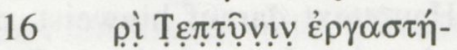

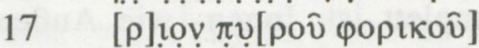

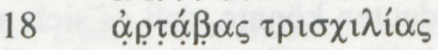

19 Spuren

20 Spuren

$21 \quad \kappa \quad$ Spuren

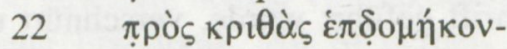

$23 \tau \alpha,(\gamma i$ ív $\tau \alpha 1)$ o.

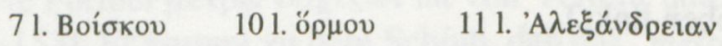

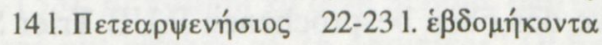

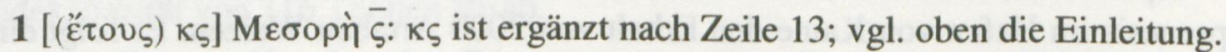
Üblicherweise stammte das in den Nauklerosquittungen angeführte Getreide von der Ernte des Jahres, in dem die Quittung auch ausgestellt wurde. Entsprechend findet sich teilweise auch statt der Angabe des Regierungsjahres, in dem das

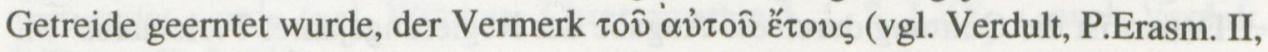
S. 127). Die Datierung wurde von derselben Hand (vgl. z.B. die Schreibung des $\mu$ 
in Z. 1 und 5) auf der scriptura interior vermerkt, dies aber wohl erst nach der Abfassung der Außenschrift.

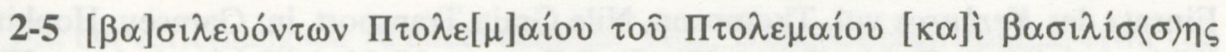

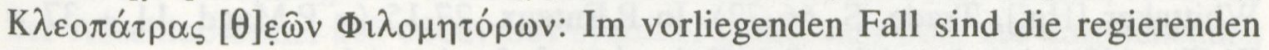
Monarchen angegeben, was normalerweise in Quittungen nicht der Fall ist. Wichtiger ist aber, daß hier das Tagesdatum fehlt. Da die Innenschrift versiegelt war, war der sichtbare Teil des Dokuments damit offiziell nicht genau datiert: das Jahresdatum konnte nur aus Z. 13 erschlossen werden.

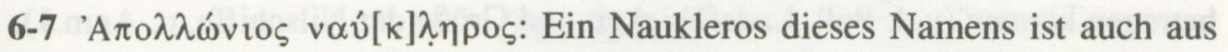
P.Erasm II 44,3 (151 v.Chr.) und 47,12 (Mitte des 2. Jh. v.Chr.) bekannt. Ebenso ist in P.Tebt. III 2, 1035,6 ein Naukleros namens Apollonios (PP V 14017; Mitte 2. Jh. v.Chr.) belegt. $\mathrm{Zu}$ den ersten beiden bemerkt Verdult, daß es keinen Grund gäbe, sie miteinander zu identifizieren (P.Erasm. II, S. 70, Anm. 12). In Anbetracht der Tatsache, daß der Name Apollonios in dieser Zeit sehr beliebt war, ist es sicherlich richtig, Vorsicht walten zu lassen. Die zeitliche und auch räumliche Nähe der Belege, von der Ausübung des Berufes einmal ganz abgesehen, läßt mir jedoch die Gleichsetzung der Namensträger recht wahrscheinlich erscheinen.

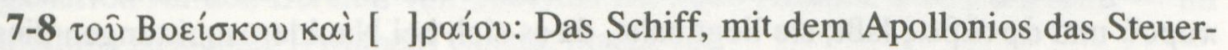
getreide verschifft hat, gehörte zwei Personen. Mit dem in den Papyri seltenen, nur in ptolemäischer Zeit belegten festlandgriechischen Namen Boiskos (siehe W. Pape, G. Benseler, Wörterbuch der griechischen Eigennamen I, Graz 1959, S. 216) ist für unseren Zeitraum meines Wissens keine weitere Person nachzuweisen (vgl. auch P.M. Fraser, E. Matthews, A Lexicon of Greek Personal Names I, Oxford 1987, S. 103).

Der Name des zweiten Schiffseigners endet im Genitiv auf - $\rho$ เıv. Die Zeilenbreite des Textes läßt zur Vervollständigung des Namens mit hoher Wahrscheinlichkeit nur noch einen Buchstaben zu. Aus den Papyri und Ostraka Ägyptens lassen sich insgesamt fünf Namen mit der verlangten Genitivendung ermitteln:

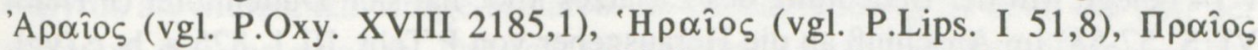
(O.Bodl. II 1845,6), T $\rho \alpha \hat{o} о \varsigma$ (O.Petr. 3223,9) und ' $\Omega \rho \alpha i ̂ \varsigma$ (O.Wilck. 1511,3). Da diese Namen jedoch nur in römischer Zeit belegt sind, möchte ich im vorliegenden Fall keine Ergänzung wagen.

$\mathrm{Da}$ sich zwei Personen das Geschäftsrisiko bzw. den finanziellen Aufwand einer Schiffseignerschaft geteilt haben, ist nach Auskunft der Papyri durchaus vorgekommen; selbst drei Eigentümer eines Frachtkahnes sind belegt (vgl. die Liste der Eigentümer bei Verdult, P.Erasm. II, S. 68-70).

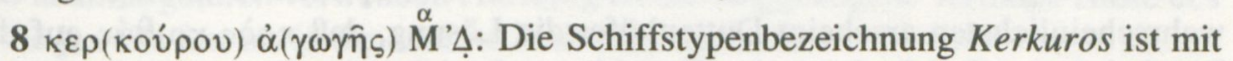
dem hierfür üblichen Monogramm geschrieben: Der zweite und der dritte Buchstabe des Wortes sind in das $\kappa$ hineingeschrieben (vgl. die Beschreibung bei Duttenhöfer, P.Heid. VI, S. 56; Verdult, P.Erasm. II, S. 156). Die Angabe $\alpha(\gamma \omega \gamma \eta ́)$ ist ebenso, wie in P.UB Trier S 84-1,4 (Nr. 1) beschrieben, abgekürzt. Der Kerkuros ist mit einer Kapazität von 1000 bis 18000 Artaben das größte und 
damit auch das meist genutzte Niltransportschiff. Am häufigsten belegt ist der Kerkouros von 10000 Artaben, was umgerechnet ca. 306,5 Tonnen sind (zum Einsatz des Kerkuros vgl. Thompson, Nile Grain Transport, in: Garnsey, Hopkins, Whittaker [Hg.], Trade, S. 66-72). In P.Enteux. 27,15 (= P.Magd. $11+37=$ W.Chr. 442) wird ein solches Schiff als $\mu \varepsilon ́ \gamma \alpha \pi \lambda$ oîov bezeichnet.

Unser Kerkuros hatte eine Ladekapazität von 14000 Artaben. Belegt ist diese Kapazität für ein solches Schiff noch nicht, doch fügt sich die Angabe gut in die anderen überlieferten Tonnagen ein, die 11000, 12000, 16000 und 18000 Artaben betragen können (vgl. Poll, Ladefähigkeit und Größe der Nilschiffe, o. Anm.1).

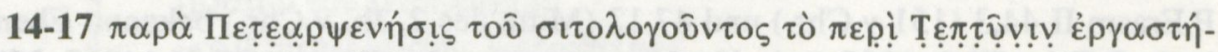

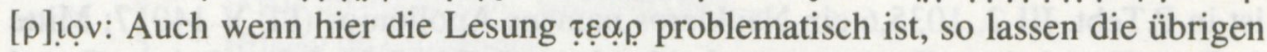
gut zu entziffernden Buchstaben kaum eine andere Komplettierung des Namens zu. Wir haben hier wohl den schon aus dem oben untersuchten P.UB Trier 84-1 (Nr. 1) bekannten Sitologen aus dem Ergasterion von Tebtynis vor uns.

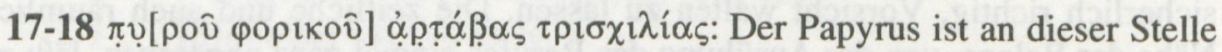
durch einen Bruch zerstört worden, so daß der Text fast vollständig abgerieben ist. Die Ergänzung von форıко v̂ ist durch den Platz bedingt, den die lacuna zwischen $\pi \cup \rho \circ \hat{~ u n d ~} \alpha \rho \tau \alpha \dot{\beta} \beta \alpha_{\varsigma}$ läßt; sie liegt nahe, da es sich bei Nauklerosquittungen dieser Art immer um Steuergetreide handelt, was dann eben teilweise auch als solches näher bezeichnet wird (s.o. zu P.UB Trier 84-1,13-15 und vgl. Verdult, P.Erasm. II, S. 127).

Was auf die Maßangabe von 3000 Artaben in den nächsten drei Zeilen folgt, ist nicht mehr lesbar. Zu erwarten ist zunächst die Angabe ( $\gamma$ ívov $\tau \alpha \mathrm{l}$ ) ' $\Gamma$, woran sich üblicherweise der Hinweis auf den rechten Gebrauch des Maßes anschließt. Die noch erhaltenen Schriftspuren lassen dies aber nicht mehr erkennen.

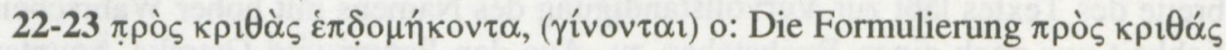
ist nur noch in P.Heid. VI 369,12; P.Tebt. III 1, 825 a, b, c und P.Münch. III 57, v 14 belegt. Mit der Bedeutung des Zusatzes $\pi \rho o ́ \varsigma$ hat sich Duttenhöfer (P.Heid. VI, S. 57-58) im Anschluß an die Herausgeber von P.Tebt. III 1, 825 a, b, c (vgl. auch A.S. Hunt, J.G. Smyly, P.Tebt. III 1, S. 333) intensiv beschäftigt. Zum einen

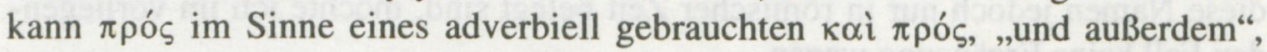
verstanden werden, was bedeuten würde, daß 3000 Artaben Weizen und 70 Artaben Gerste geladen worden sind. Hierzu bemerkt Duttenhöfer aber zu Recht, daß die 70 Artaben Gerste dann auch in der Innenschrift Erwähnung gefunden

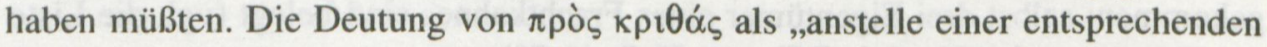
Gerstenmenge" ist wohl unwahrscheinlich und m.E. sprachlich unmöglich. Am

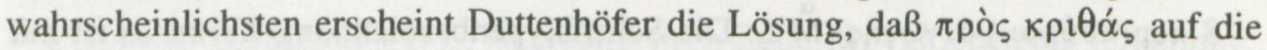
Reinheit bzw. die Mischung des Getreides zu beziehen ist. Das würde bedeuten, daß das Getreide, das Apollonios geladen hatte, eine Beimischung von 70 Artaben Gerste auf 3000 Artaben Weizen hatte, somit also einen Anteil von 2,33\% Gerste enthalten hat. In der lacuna wären dann neben der Angabe über den rechten 
Gebrauch des Maßes auch noch der Vermerk über den Reinheitsgrad des verladenen Getreides zu erwarten.

Mit der Ziffernangabe 70 endet dann der Text. Weitere Schriftspuren, etwa von

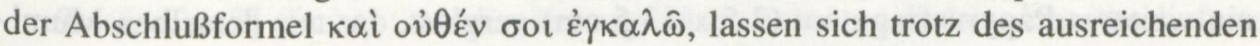
Platzes nicht ausmachen; die Formel ist aber auch nicht obligatorisch (vgl. die Bemerkung von Verdult, P.Erasm. II, S. 129).

\section{Übersetzung}

26. Regierungsjahr, 6. Mesore, 3000 Artaben Weizen.

Unter der Königsherrschaft des Ptolemaios, des Sohnes des Ptolemaios, und der Königin Kleopatra, der Mutterliebenden Götter. Es erklärt Apollonios, Naukleros des Kerkuros von Boiskos und [-]raios mit einer Ladekapazität von 14000 (Artaben), geladen zu haben im Hafen von Kaine mit dem Ziel Alexandria, Königlicher Speicher, von den Erträgen des 26. Jahres von Peteharpsenesis, der Sitologe im Ergasterion für den Bereich von Tebtynis ist, 3000 Artaben Steuerweizen, --- im Verhältnis zu siebzig (Artaben) Gerste, macht 70.

\section{Quittung des NN für Pe[teharpsenesis]}

P.UB Trier S 84-3b

Kaine (Arsinoites)

\author{
$4,5 \times 16,5 \mathrm{~cm}$ \\ Tafel IV
}

Mitte 2. Jh. v.Chr. Recto, Verso unbeschrieben

Der Papyrus ist links abgebrochen, rechts reicht die Beschriftung bis zum Rand. Durch den Bruch fehlt die linke Hälfte des Textes. Die Rundung des oberen Randes, die in den rechten Rand übergeht, läßt sich mit der Zweitverwendung des Papyrus als Mumienkartonage erklären. In dieser Rundung ist noch ein Teil der scriptura interior erhalten. Da sich am unteren Rand noch die „Nichtangriffs-

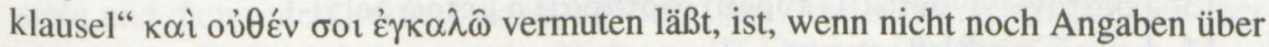
den Verfasser des Schriftstückes folgten, zumindest der komplette Umfang der Quittung erhalten. Das Verso ist unbeschrieben.

Die Hand läßt sich ebenso wie die der beiden anderen Papyri in die Mitte des 2. Jhs. v.Chr. datieren, also in die Herrschaft Ptolemaios' VI. und Kleopatras II. Der Schreiber hat die einzelnen Buchstaben klar abgetrennt, deutlich geschrieben und kaum Ligaturen verwendet. Auffällig ist die langgezogene vertikale Haste des $\rho$, die jeweils fast bis in die nächste Zeile hinunterreicht.

Ein Naukleros einer Prosagogis von 2900 Artaben Ladekapazität, dessen Name verloren ist, hat von einem Sitologen, der wahrscheinlich Peteharpsenesis heißt und für das Ergasterion von Tebtynis zuständig ist, im Hafen von Kaine 100 Artaben Weizen übernommen, die er nach Alexandria verschiffen wird. Trotz des schlechten Erhaltungszustandes läßt sich der Text aufgrund des reichhaltigen Par- 
allelmaterials rekonstruieren. Er folgt insgesamt den Parallelen und bietet des weiteren keine Besonderheiten.

Zusammen mit diesem Symbolon ist unter der Inventarnr. P.UB S 84-3b noch ein weiteres Papyrusfragment $(2,5 \times 13,5 \mathrm{~cm})$ verglast, das aus derselben Mumienkartonage stammt. Ob beide Papyrusfragmente von derselben Hand stammen, läßt sich m.E. nicht sagen, da zu wenig an Schrift auf dem zweiten Fragment erhalten ist. Zwar sieht die Schrift auf den ersten Blick ähnlich aus, doch vergleicht man z.B. das $\kappa$ in Z. 6 des ersten Textes mit dem $\kappa$ in Z. 2 des kleinen Fragments, so sind diese einander eher unähnlich. Noch auffälliger ist die unterschiedliche Schreibung des 1 , das in dem mitverglasten Fragment mit einem Strich leicht diagonal von links oben nach rechts unten gezogen wurde (Z. 4), wohingegen es in der Nauklerosquittung leicht nach rechts geneigt ist (Z. 6). Die auf dem Fragment lesbaren Schriftspuren lassen sich schließlich kaum mit dem gut zu rekonstruierenden Vertragstext der Nauklerosquittung in Übereinstimmung bringen.

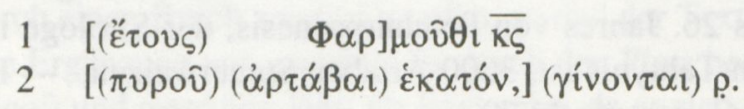

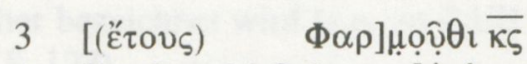

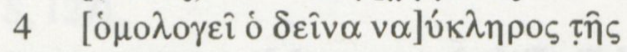

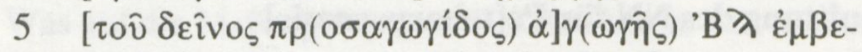

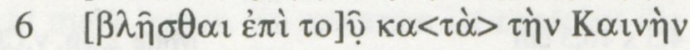

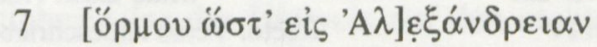

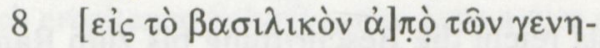

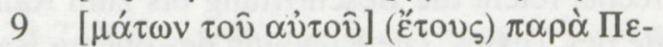

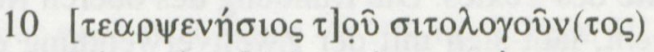

11 [

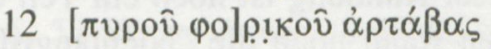

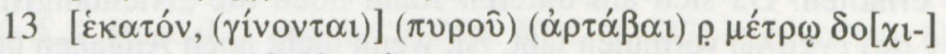

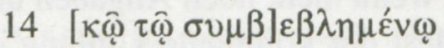

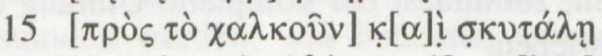

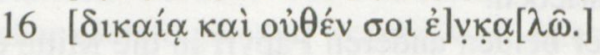

161. $\dot{\varepsilon} \gamma \kappa \alpha \lambda \hat{\omega}$

$1 \Phi \propto \rho] \mu \circ \hat{v} \theta \imath \overline{\kappa \varsigma}$ : Die Lesung $\overline{\kappa \varsigma}$ ist durch den Abrieb, den die Schriftzeichen teilweise an der Papyrusoberfläche erlitten haben, schwierig, doch läßt sie sich durch Z. 3, in der ebenfalls die Datierung steht, stützen.

$5 \pi \rho(o \sigma \alpha \gamma \omega \gamma i \delta \circ \varsigma) \dot{\alpha}] \gamma(\omega \gamma \eta \hat{\varsigma} \varsigma)$ 'B $\lambda$ : Die Rekonstruktion der Schiffstypenbezeichnung $\pi \rho \circ \sigma \alpha \gamma \omega \gamma$ is ist durch die Frachtkapazität von 2900 Artaben als sicher 
anzusehen. Zum einen gibt es hierfür in P.Erasm. II fünf Parallelbeispiele (P.Erasm. II 38,4; 41,5; 46,4; 49,4; 53,5), zum anderen enthält auch der oben behandelte P.UB Trier S. 84-1 (Nr. 1) diese Angabe.

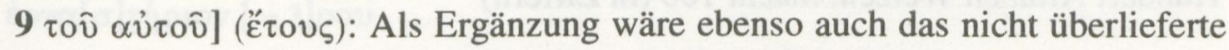
Regierungsjahr denkbar (vgl. z.B. P.Erasm. II 28,4; 30,4; 32,4).

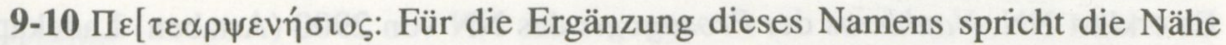
des Papyrus zu Nr. 1 und 2. Alle drei stammen aus derselben Kartonage, aus derselben Zeit und demselben Hafen.

$11 T \varepsilon] \pi \tau \underline{v} v \underline{v}$ : Die Lesung ist problematisch. Die Angabe des Hafens von Kaine ist hier sicher gelesen ist. Der Hafen wird nach den Zeugnissen bisher nur noch aus dem Ergasterion von Oxyrhyncha und eventuell von Anubias mit Steuergetreide beliefert (Verdult, P.Erasm. II, S. 3; Duttenhöfer, P.Heid. VI, S. 56-57). Für diese beiden in Frage kommenden Ergänzungen bieten die Schriftspuren keinen Hinweis, wohingegen die Lesung - $\pi \tau \hat{v} v ı$ veher mit ihnen übereinstimmt.

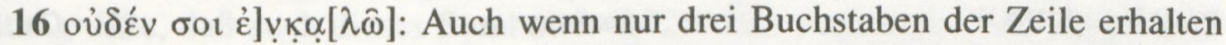
sind, so fügt sich an dieser Stelle die Nichtangriffsklausel perfekt in den Vertragskontext ein. Es ist nicht zu bezweifeln, daß sie hier gestanden hat. Der Naukleros bescheinigt dem Sitologen mit dieser Aussage, daß das von ihm übernommene Getreide den in der Urkunde festgesetzten Zustand hatte und er diesbezüglich keine Rechtsmittel mehr gegen ihn einlegen konnte. Das bedeutet auch, daß im Zielhafen bei einem eventuellen Fehlen von Getreide der Naukleros dafür haftete (vgl. Verdult, P.Erasm. II, S. 153). Diese Klausel, mit der sich der Naukleros in eine für ihn ungünstige Rechtsposition brachte, diente wohl dazu, den Staat „prozessrechtlich zu Lasten des Schiffers“ abzusichern (Meyer-Termeer, Haftung, S. 57). Damit trägt der Naukleros also die Transportgefahr; er hat eine Stellung, die der des Steuerpächters ähnelt (vgl. W. Kunkel, Verwaltungsakten aus spätptolemäischer Zeit, APF 8, 1927, 185).

Verdult weist zurecht darauf hin, daß das Verb $\dot{\varepsilon} \gamma \kappa \alpha \lambda \hat{\omega}$ in der ersten Person steht und damit nicht mit der Form ó $\mu о \lambda о \gamma \varepsilon \hat{~ a m ~ B e g i n n ~ d e s ~ V e r t r a g s t e x t e s ~ u ̈ b e r-~}$ einstimmt. Dadurch bekommt die Nichtangriffsklausel „an additional emphasis“ (P.Erasm. II, S. 127-128). Ihm zufolge ist die Verwendung der ersten Person Singular auch als Hinweis darauf zu werten, daß die Quittung von dem betreffenden Naukleros selbst geschrieben wurde (P.Erasm. II, S. 128; zur Nichtangriffsklausel allgemein vgl. H.-A. Rupprecht, Studien zur Quittung im Recht der graecoägyptischen Papyri, München 1971, S. 96-103).

Die Formel könnte vielleicht ursprünglich in die Unterschrift des Naukleros gehört haben. Wenn dies der Fall wäre, dann läge mit diesem Papyrus eine Abschrift der Originalquittung vor, da der Text insgesamt von einer Hand ausgeführt worden ist. 


\section{Übersetzung}

x. Jahr, 26. Pharmuthi.

Hundert Artaben Weizen, macht 100 (in Ziffern)

x. Jahr, 26. Pharmuthi.

Es erklärt NN, Naukleros der Prosagogis des NN von 2900 (Artaben) Ladekapazität, im Hafen von Kaine mit dem Ziel Alexandria, Königlicher Speicher, von den Erträgen des (gegenwärtigen) Jahres von Peteharpsenesis, dem Sitologen im Ergasterion für den Bereich von Tebtynis, hundert Artaben Steuerweizen, macht 100 Artaben Weizen, mit dem Empfangsmaß, das verglichen ist mit dem bronzenen Normalmaß und mit dem Streichmaß, geladen zu haben. Und ich werde keine Forderungen gegen dich geltend machen.

\section{Quittung des NN für Peteharpsenesis}

P.UB Trier S 108-4

Kaine (Arsinoites) ?
$6,5 \times 8 \mathrm{~cm}$

Tafel V
153 v.Chr. (?)

Verso; Recto: Tintenspuren

Das Papyrusfragment, das 1998 erworben wurde, läßt seiner Form nach eindeutig die Zweitverwendung als Mumienkartonage erkennen: Es war zu einem Fußteil zurechtgeschnitten worden. Die erhaltenen Reste der Nauklerosquittung laufen quer zu den Fasern, doch oberhalb des Textes verläuft eine zweite Schicht waagerecht verlaufender Fasern, so daß es sich wohl um das Anfangsstück einer Papyrusrolle handelt, das sogenannte Protokoll. Die andere, qualitativ bessere Seite mit den zum Text parallel verlaufenden Fasern trägt Schriftspuren, die von einer Datierung herrühren dürften.

Der Text, der ebenso wie die Texte der zuvor besprochenen Papyri in einer geübten Geschäftsschrift des 2. Jh. v.Chr. geschrieben wurde, läßt sich eindeutig dem Formular einer Nauklerosquittung zuweisen, die für einen Mann namens Peteharpsenesis ausgestellt wurde und auf ein 28. Regierungsjahr datiert ist. Über dem Haupttext, der Außenschrift, ist noch ein Rest der Innenschrift zu sehen. Auffällig an diesem Papyrus ist, daß die Quittung nicht wie sonst üblich im Hoch-, sondern im Querformat abgefaßt worden ist.

Der Name des Sitologen und die Datierung ins 2. Jh. v.Chr. könnten darauf hinweisen, daß wir hier eine weitere Quittung vorliegen haben, die im Hafen von Kaine für Peteharpsenesis, den Verwalter des Ergasterions von Tebtynis, ausgestellt worden ist.

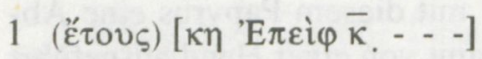

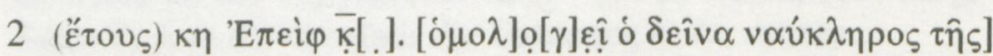

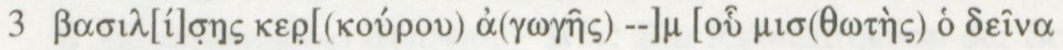
$\dot{\varepsilon} \mu \beta \varepsilon \beta \lambda \hat{\eta} \sigma \theta \alpha l]$ 


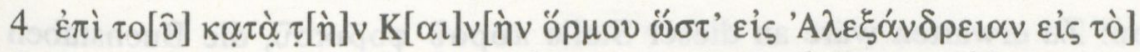

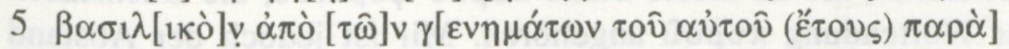

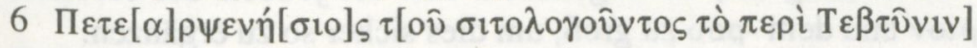

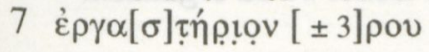

Rückseite:

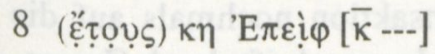

31. $\beta \alpha \sigma \imath \lambda i ́ \sigma \sigma\rangle$

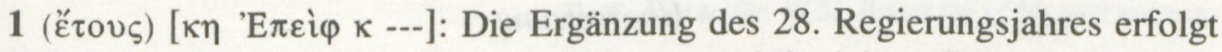
nach Z. 2, in der es sicher zu lesen ist. Wie gesagt, läßt sich vorliegende Quittung paläographisch ebenso wie Nr. 1-3 dem 2. Jh. v.Chr. zuweisen. Da es sich des weiteren um denselben Sitologen namens Peteharpsenesis handelt, liegt die Vermutung nahe, daß auch dieses Symbolon im 28. Jahr Ptolemaios' VI. ausgestellt worden ist, und zwar Ende des Monats Epeiph, da das $\kappa$ in der Tagesdatierung noch relativ gut zu lesen ist. Die Quittung wurde somit wahrscheinlich Mitte bis Ende August 153 v.Chr. ausgestellt.

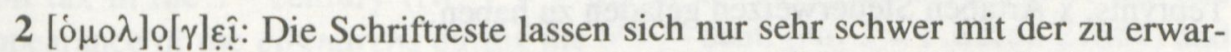
tenden „Homologieformel“ übereinbringen, doch muß das ergänzte Verb hier aufgrund des Gesamtkontextes mit einiger Sicherheit gestanden haben.

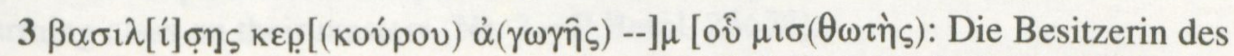
Frachtkahnes war die Königin, wie dies auch in P.Lille I 23; P.Erasm. II 30; 39; 42; 43; P.Tebt. III 2, 1034; P.Sorb. inv. 110a der Fall ist (vgl. die Zusammenstellung der Schiffseigner bei Verdult, P.Erasm. II, S. 68-70). Ob es sich tatsächlich um einen Kerkuros gehandelt hat, ist nicht sicher, genauso wäre eine Ergän-

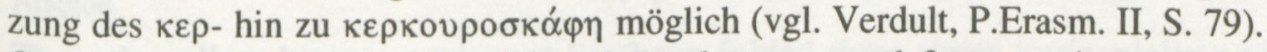
Gerade wenn ein Schiff der Königin gehörte, kam es vor, daß es von einer anderen Person gemietet wurde (P.Erasm. II 30; 42), die es dann dem Naukleros zur Verfügung stellte. Mieter und Naukleros müssen nicht zwingend dieselben Personen sein (Verdult, P.Erasm. II, S. 76). Aus Platzgründen vermute ich, daß die Angabe des Mieters im vorliegendem Papyrus gestanden hat.

$4 \mathrm{~K}\left[\alpha_{1}\right] v[\grave{\eta} v$ ó $\rho \mu o v$ : Die Ergänzung von Kaine (vgl. den Kommentar zu Nr. 1, Z. 4-6) ist keineswegs sicher, doch aufgrund des oben dargelegten Gesamtkontextes der Quittung sehr wahrscheinlich.

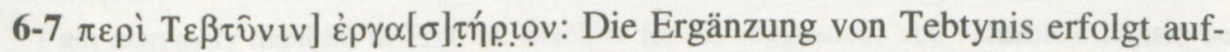
grund der Parallelen Nr. 1-3, die bezeugen, daß der Sitologe Peteharpsenesis für diesen Ort zuständig war. 
7 könnten also der Wortendung $\pi v \rho \circ \hat{v}$ angehören. Mir ist jedoch der Abstand

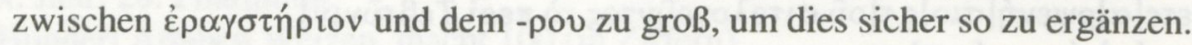

\section{Rückseite}

8 (๕ุ klären, weshalb der Verfasser das Datum der Transaktion nochmals auf die „Rückseite“ der Quittung geschrieben hat, wenn die Innenschrift durch Zusammenrollen versiegelt wurde.

\section{Übersetzung}

28. Jahr, $20+x$. Epeiph

28. Jahr, $20+x$. Epeiph. Es erklärt NN, Naukleros des Kerkuros der Königin von $x$ (Artaben) Ladekapazität, den gemietet hat NN, im Hafen von Kaine mit dem Ziel Alexandria, königlicher Speicher, von den Erträgen des gegenwärtigen Jahres von Peteharpsenesis, dem Sitologen des Ergasterions für den Bereich von Tebtynis, x Artaben Steuerweizen geladen zu haben...

28. Jahr, $20+x$. Epeiph 


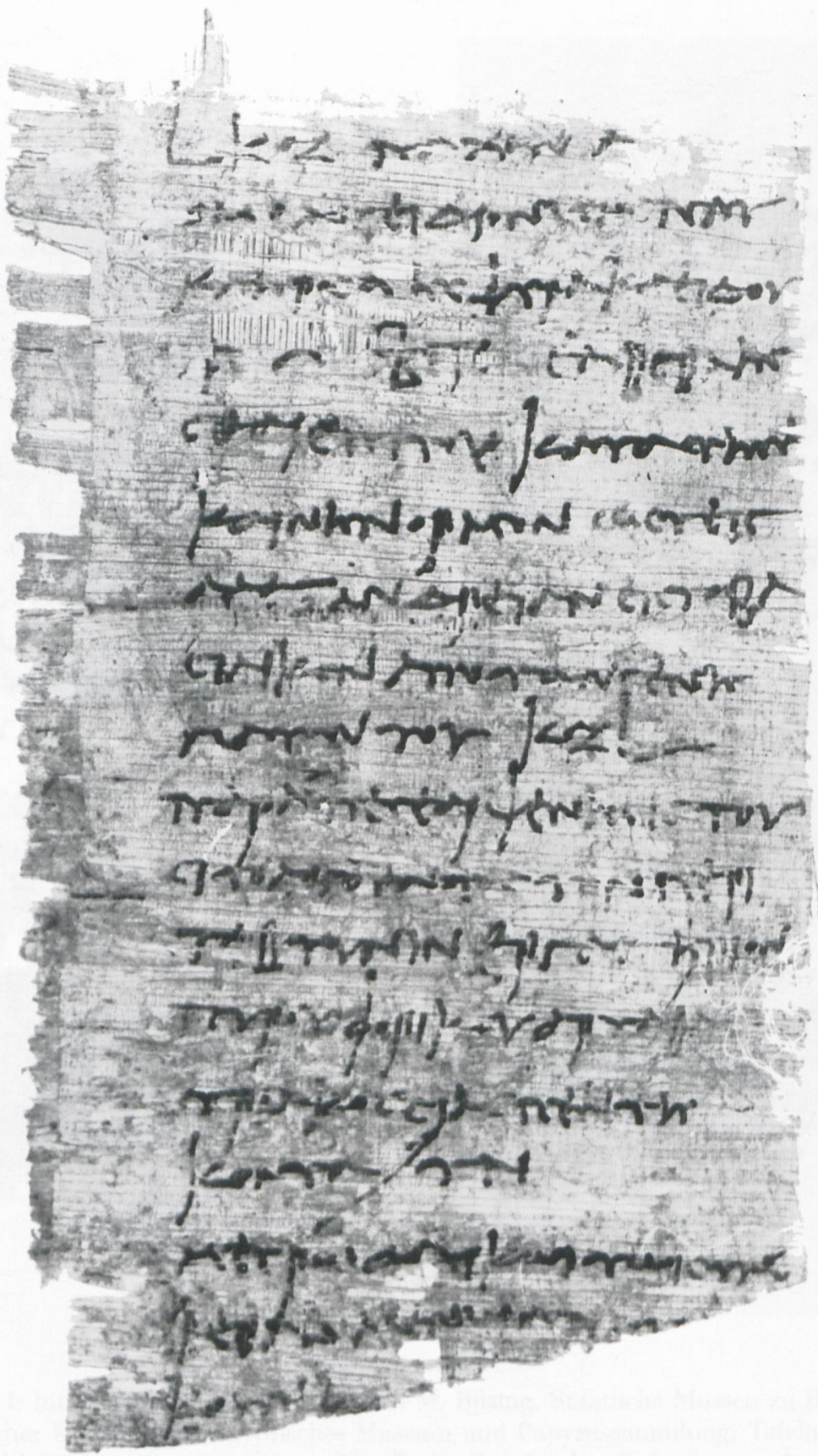

Nauklerosquittung (P.UB Trier S 84-1); $\mathrm{zu}$ : St. Pfeiffer, S. 25 ff., Nr. 1 
TAFEL III

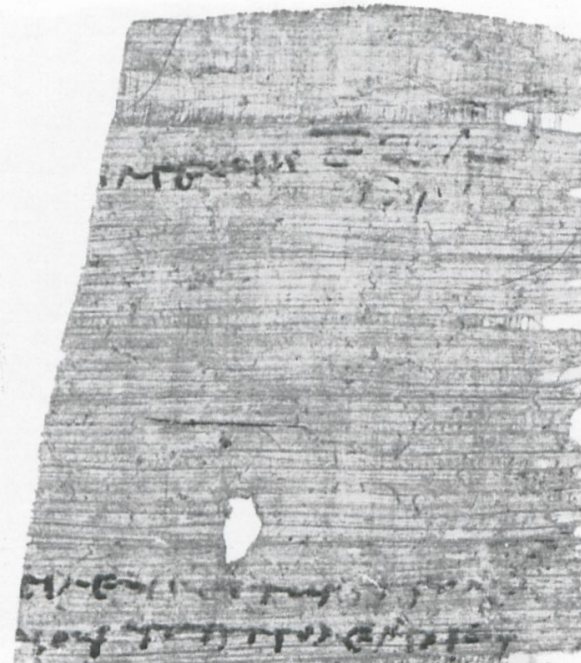

Bselachekecton the

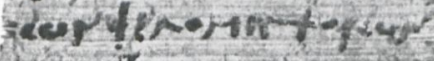

- A. Fy

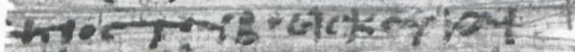

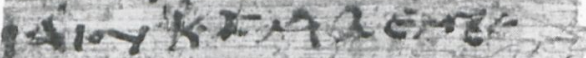

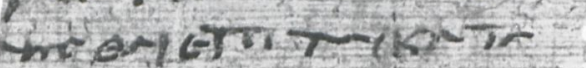

h.

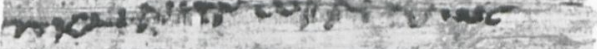

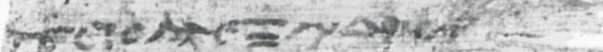

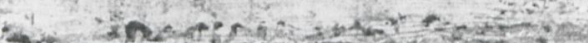

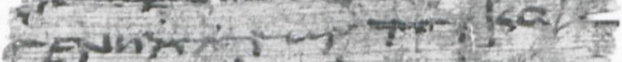

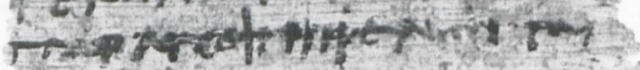
(n) r s whenen

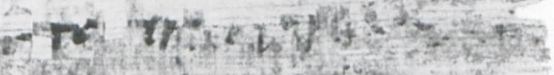
in

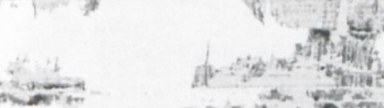

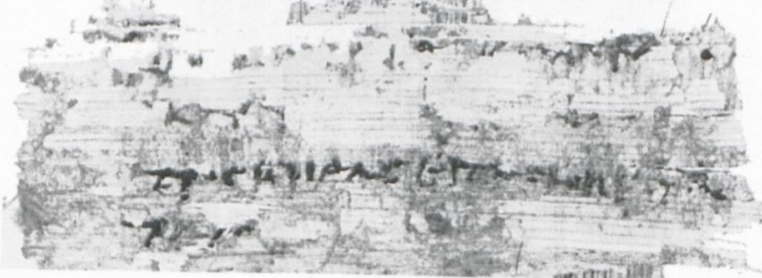

Nauklerosquittung (P.UB Trier S 84-2); zu: St. Pfeiffer, S. 25 ff., Nr. 2 
TAFEL IV

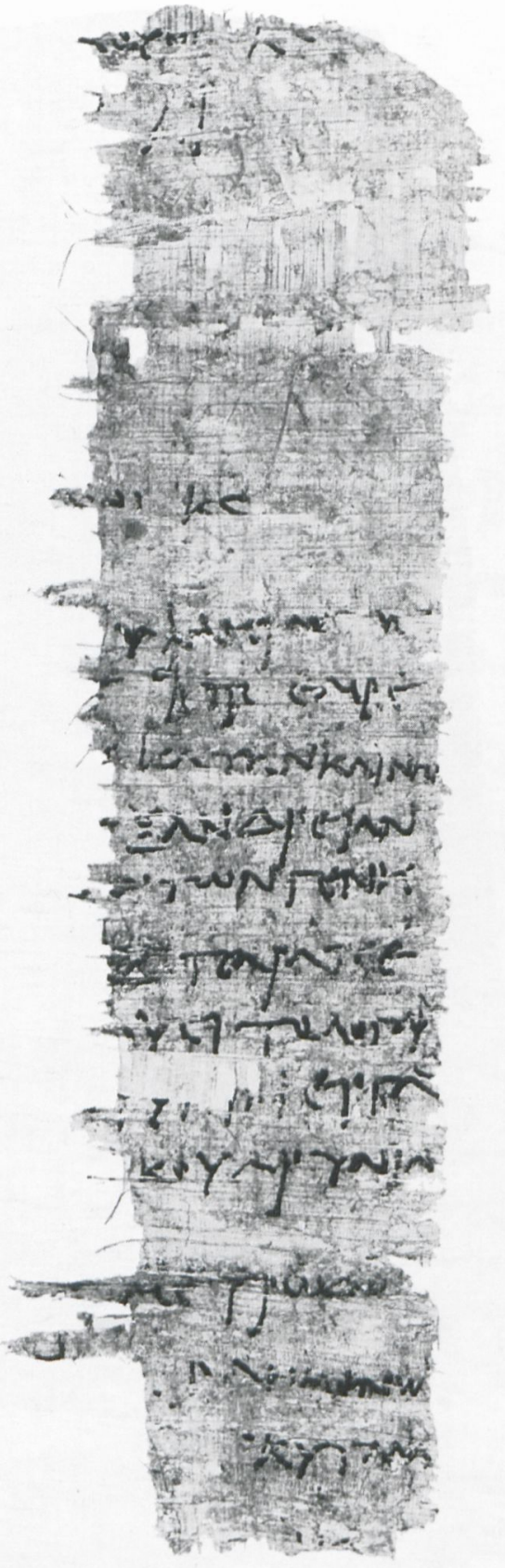

Nauklerosquittung (P.UB Trier S 84-3b); zu: St. Pfeiffer, S. 25 ff., Nr. 3 
TAFEL V

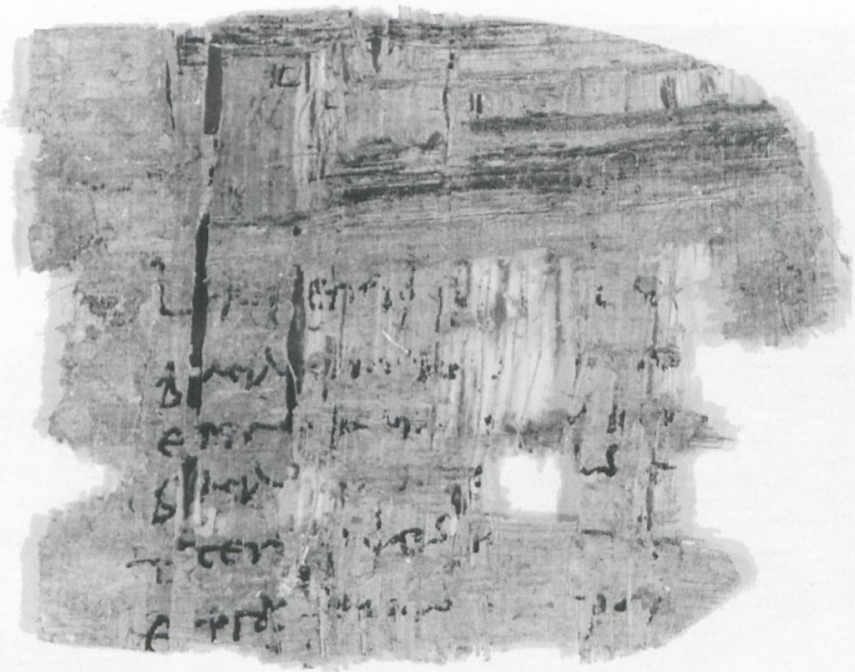

Nauklerosquittung (P.UB Trier S 108-4);

zu: St. Pfeiffer, S. 25 ff., Nr. 4

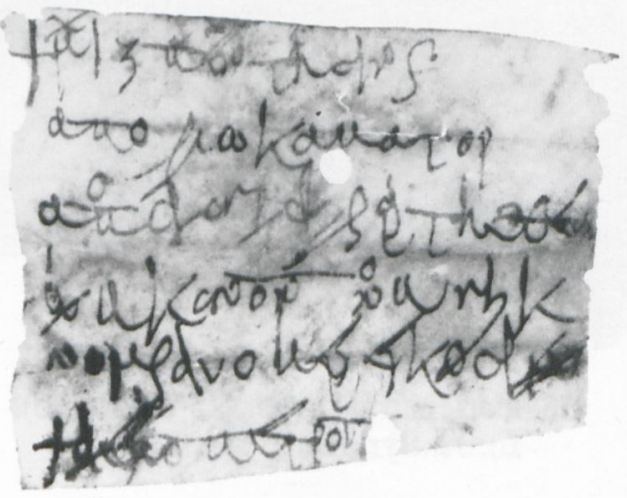

Steuerquittung (P.Berol. 25118);

zu: A. Syrkou, S. 43 f., Nr. 1 